\title{
Optimisation of the Orchestra Pit Acoustics in Opera Houses by Acoustical Simulations using the Finite Element Method
}

\author{
Jan Michael Kimmich ${ }^{1}$, Anton Schlesinger ${ }^{2}$, Martin Ochmann ${ }^{2}$ and Stefan Frank O,* $^{1,}$ \\ ${ }^{1}$ Hochschule für Technik und Wirtschaft Berlin, FB 2 - Engineering, Germany \\ ${ }^{2}$ Beuth Hochschule für Technik Berlin, Projektgruppe Computational Acoustics, Germany
}

\begin{abstract}
Musicians in the orchestra pit in opera houses are exposed to relatively high sound pressure levels during the rehearsals and the performance. As a consequence, the communication between the diverse musicians is disturbed and potentially the hearing ability is endangered. In order to improve the situation for the musicians in-situ measurements of the room acoustic properties are performed (e.g. reverberation time $\mathrm{T}$, clarity $\mathrm{C}_{80}$, strength $\mathrm{G}$ etc.) in order to provide evaluation data. On the other hand, acoustic simulations of the entire opera house or parts of it are performed by both the Finite Element Method (FEM) for low frequencies and the ray tracing approach for middle and high frequencies. The results are compared to the experimental data. Subsequently, optimization measures, like additional absorbers or diffusers, can be investigated in detail by the simulations. The most promising will eventually be chosen for realization and be measured in-situ.
\end{abstract}

\section{Introduction}

The room acoustics of opera houses is determined by three acoustically coupled rooms, namely the fly tower, the orchestra pit and the auditorium. Within the project SIMOPERA (simulation and optimization of room acoustical fields by considering the Deutsche Oper Berlin) room acoustics shall be simulated and optimized using the example of Deutsche Oper Berlin (DOB). It is necessary to improve the acoustic conditions in the orchestra pit due to high sound pressure levels that are potentially dangerous for the health of the musicians.

The simulation of sound fields in these rooms requires the application of different approaches. Simulation methods based on geometrical acoustics can be used when the wavelength of propagating sound is small in relation to the characteristic room dimension, i.e. for the auditorium large in volume. In rooms which are small with respect to the wavelength the sound field below the Schroeder frequency is dominated by modal behaviour. These effects can only be simulated by wave-based methods like the Finite Element Method (FEM) and the Boundary Element Method (BEM). Within this project the

*Corresponding author: stefan.frank@htw-berlin.de 
different simulation methods shall be applied to the DOB and subsequently be evaluated by room acoustic measurements.

The DOB was rebuilt in the early nineteen sixties after its destruction in World War II. The fan-shaped auditorium holds about 1900 seats and is inspired by the Bayreuth Festspielhaus [1]. Acoustic consulting was done by L. Cremer and documented in [2]. It is worth mentioning the acoustic treatment, especially the design of the so called Rabitz ceiling (i.e. wire frame coffering) which is designed as a staggered reflector. The acoustics of the auditorium is controlled by the suspended Rabitz ceiling, the partly perforated panel absorbers that cover the side walls, and of course the upholstered chairs. The auditorium volume is about $11000 \mathrm{~m}^{3}$, whereas the acoustically coupled orchestra pit and fly tower hold about 400 and $17000 \mathrm{~m}^{3}$, respectively [1 to 3].

Results of room acoustic measurements at DOB with iron curtain open and closed for coupling and decoupling the fly tower are presented. Furthermore, the setup and results of simulations applying both the wave based methods, such as FEM, and the geometrical acoustics methods will be introduced. The influence of low frequency absorption implemented on the boundaries of the orchestra pit is simulated. For the decoupled orchestra pit a numerical frequency analysis applying FEM method is shown for one particular set of impedance boundary conditions on the opening aperture of the orchestra pit.
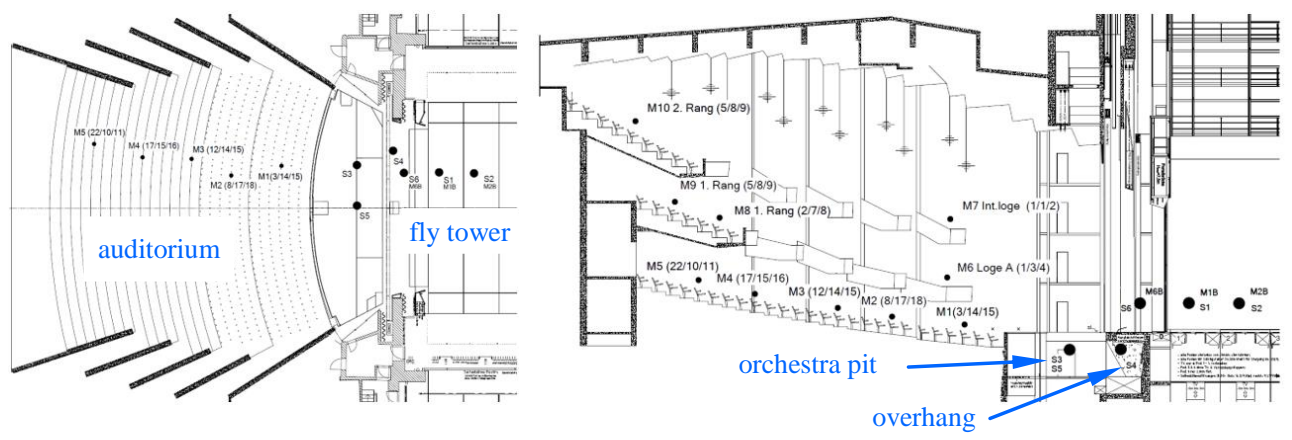

Fig. 1. Top view (left) and section (right) of the auditorium, orchestra pit and fly tower of the Deutsche Oper Berlin (DOB). Indicated are the source (S) and measurement positions (M) used for room acoustic measurements.

\section{Room Acoustic Measurements}

\subsection{Setup, Equipment and Method}

This paper emphasizes the room acoustic parameters for transparency and stage acoustics. However, results of the reverberation time and sound strength $G$ in comparison with other opera houses were originally published in [4]. For capturing the current state of room acoustics, measurements were carried out according to ISO 3382-1:2009 [5] in the absence of audience and scenery. The orchestra pit was positioned in the most frequently used setting for opera performances which is $2.9 \mathrm{~m}$ below stage level. The side walls of the empty fly tower were covered with theatre curtains to avoid the occurrence of flutter echoes. A small part of the staggered reflector under the ceiling of the auditorium was opened because the lighting bridge located there was used in the previous performances. The influence of opening this part of the ceiling on room acoustic quantities was already shown by Cremer [2] in scale model experiments. Ten measurement positions in the stalls, balconies and lodges were chosen, which are shown in Fig. 1. Impulse responses for three source positions on the stage and another three in the orchestra pit were measured. The 
omnidirectional sound source was placed at three different positions in the front and middle part of the stage. Inside the orchestra pit also three source positions were used with source position S4 lying in the so-called overhang area below the stage.

The measurement equipment consists of the sound sources Type 4292-L and QSAM Type QS-12 as dodecahedron loudspeakers and the Omnisource Type 4295 with the power amplifiers PA 1000 and Type 2734 (all Brüel \& Kjær). As a receiver, the omnidirectional microphone Earthworks Type M30 with the RME Type interface is used. A logarithmic sweep has been used as the excitation signal. It is applied three times per source-receiver combination for increasing the SNR of the measurements.

\subsection{Analysis of Room Acoustics}

Measurements of the reverberation time were performed for three different configu-rations. Usually the $\mathrm{T}_{30}$ method is applied from the one-third octave band $160 \mathrm{~Hz}$ upwards. However, at low frequencies, the $T_{20}$ method had to be applied for reasons of an otherwise too low SNR. Firstly, the reverberation time was measured with closed iron curtain and source and receiver in the orchestra pit (CC). Secondly, with iron curtain closed, sound sources in the orchestra pit and receivers on measurement positions M1 to M10 as indicated in Fig. 1. Thirdly, with iron curtain open, receivers on positions M1 to M10 and sound sources on the stage and in the orchestra pit. The mean values are given in Fig. 2 for onethird octave bands together with the median and 25 th as well as 75 th percentiles.

From the increasing percentile bars below $250 \mathrm{~Hz}$, it can be seen that there is a dependency of reverberation time on the measurement positions. This variation of the reverberation time with different positions is a characteristic property of coupled rooms. For the configuration with the iron curtain opened which couples the fly tower large in volume, the results $(\mathrm{CO})$ vary even more. The linear regression applied to the logarithmic energy decline for calculating the reverberation time as described in [5] implicates uncertainty, since the decline is not linear in coupled rooms $[3,6]$.
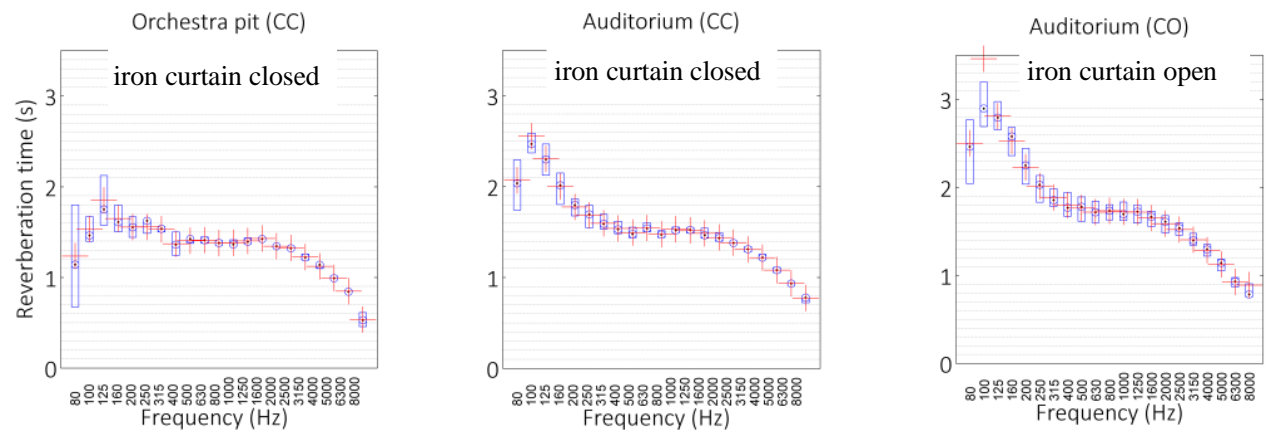

Fig. 2 Reverberation time vs. frequency in one-third octave bands for three different measurement positions: In the orchestra pit with the iron curtain closed (CC, left), in the auditorium with the iron curtain closed (CC, middle) and in the auditorium with the iron curtain open ( $\mathrm{CO}$, right). Median is marked "o", mean value "+"

In order to determine the transparency of sound in the auditorium a set of three well established room acoustic parameters have been calculated, namely clarity $\mathrm{C}_{80}$, definition $\mathrm{D}_{50}$ and center time $\mathrm{T}_{\mathrm{C}}$ [5]. For clarity and definition a common tendency with higher values towards rear listener positions has been found. The highest values for both 
parameters were measured at position M9 on the first balcony which benefits from early reflections of the ceiling under the second balcony floor. Slightly lower are the parameters for position M10 which benefits from proximity to the sidewalls in the rear part of the auditorium as well as from the reflecting ceiling. The averaged values over the ten measurement positions inside the auditorium are given in Table 2.1. The measured parameters shown here are well within the range described in [5].

Table 2.1: Room acoustic parameters for transparency in the auditorium with iron curtain open (CO); ten measurement positions and source positions on the stage and in the orchestra pit. The result is given as mean value \pm standard deviation

\begin{tabular}{|c|c|c|}
\hline Clarity $\mathbf{C}_{\mathbf{8 0}}$ in $\mathbf{~ d B}$ & Definition $\mathbf{D}_{\mathbf{5 0}}$ in $\mathbf{~ d B}$ & Center time $\mathbf{T}_{\mathbf{c}}$ in $\mathbf{~ m s}$ \\
\hline$-0.43 \pm 0.33$ & $+0.33 \pm 0.02$ & $+130 \pm 3.4$ \\
\hline
\end{tabular}

As a measure of the room acoustic support on stages for musicians, Early Support $\mathrm{ST}_{\text {early }}$ was measured. Extensive work was done in the field of stage acoustics which comprises research on physical room acoustic measures as $\mathrm{ST}_{\text {early }}$ and their correlation with perceptual evaluations by musicians e.g. by Gade [8] and Dammerud [9]. However, less attention is paid to the situation in orchestra pits where one has to minimize sound exposure for musicians and simultaneously ensure mutual audibility within the orchestra. For assessing stage acoustics inside the orchestra pit, $\mathrm{ST}_{\text {early }}$ was measured. The respective values are $\mathrm{ST}_{\text {early }}=-9.3,-7.3$ and $-10.8 \mathrm{~dB}$ at three positions S3, S4 and S5. As might be expected the highest value can be found in the overhang at S4. Again, these values lie well within the range of comparable measurements in orchestra pits [10]. Furthermore, frequency averaged values for the Early Decay Time EDT have been published in [4].

\section{Room Acoustic Simulations}

\subsection{FEM Frequency Analysis of the Decoupled Orchestra Pit}

For the development of acoustic treatment which can improve the acoustic conditions in the orchestra pit, room acoustic simulations were carried out. There are two different objectives for the simulations within the project: Firstly the low frequency sound field inside the orchestra pit has to be calculated to develop selective room acoustic treatment in order to control the room modes. Secondly, the effect of constructional changes inside the orchestra pit on the radiation of sound into the coupled auditorium has to be assessed. For the first task the use of wave-based simulation methods, i.e. FEM is suitable. The second part makes use of geometrical acoustics since the volume of the auditorium - given the available computing power of today - is too large to be simulated with wave-based methods.

In small rooms like the orchestra pit the low frequency sound field beneath the Schroeder frequency is dominated by the existence of well separated room resonances [6]. For a complex shaped room there is no analytical solution, and therefore the application of the Finite Element Method (FEM) for natural frequency analysis is appropriate. The limiting criterion is that each wavelength has to be discretized with at least six elements in order to avoid numerical dispersion. For a cube with a volume of $12000 \mathrm{~m}^{3}$ this means that at least $6 \cdot 10^{4}$ hexahedral cells at $100 \mathrm{~Hz}, 6 \cdot 10^{7}$ cells at $1 \mathrm{kHz}$ and $6 \cdot 10^{10}$ cells at $10 \mathrm{kHz}$ have to be used. The necessary cell number increases with the power of three with decreasing wavelength. Thus the FEM method is currently limited to relatively low 
frequencies for large opera house volumes. On principle, the FEM is not suitable for dealing with so called exterior problems, as is the case for the open orchestra pit. Several methods for treating exterior problems with the FEM exist [11]. Here, for first calculations with COMSOL Multiphysics 5.3a the plane wave boundary condition $Z=\rho_{0} \mathrm{c}$ has been applied to the opening aperture as shown in Fig. 3. A detailed description of the orchestra pit and the setup of the FEM simulation can be found in [4]. As it can be seen from the comparison of sound pressure distributions on the top boundary, the damping is the more efficient the higher the natural frequency. Since $Z=\rho_{0} c$ is the impedance in the far field i.e. for plane waves this approach seems not to work well for low frequencies. This can be seen in the top left plot of Fig. 3 for $\mathrm{f}=(18.6+13.5 \mathrm{i}) \mathrm{Hz}$ where high sound pressures on the top boundary occur. In contrast to the bottom right plot for $\mathrm{f}=(76.5+0.8 \mathrm{i}) \mathrm{Hz}$ sound pressures on the top boundary are considerably lower.

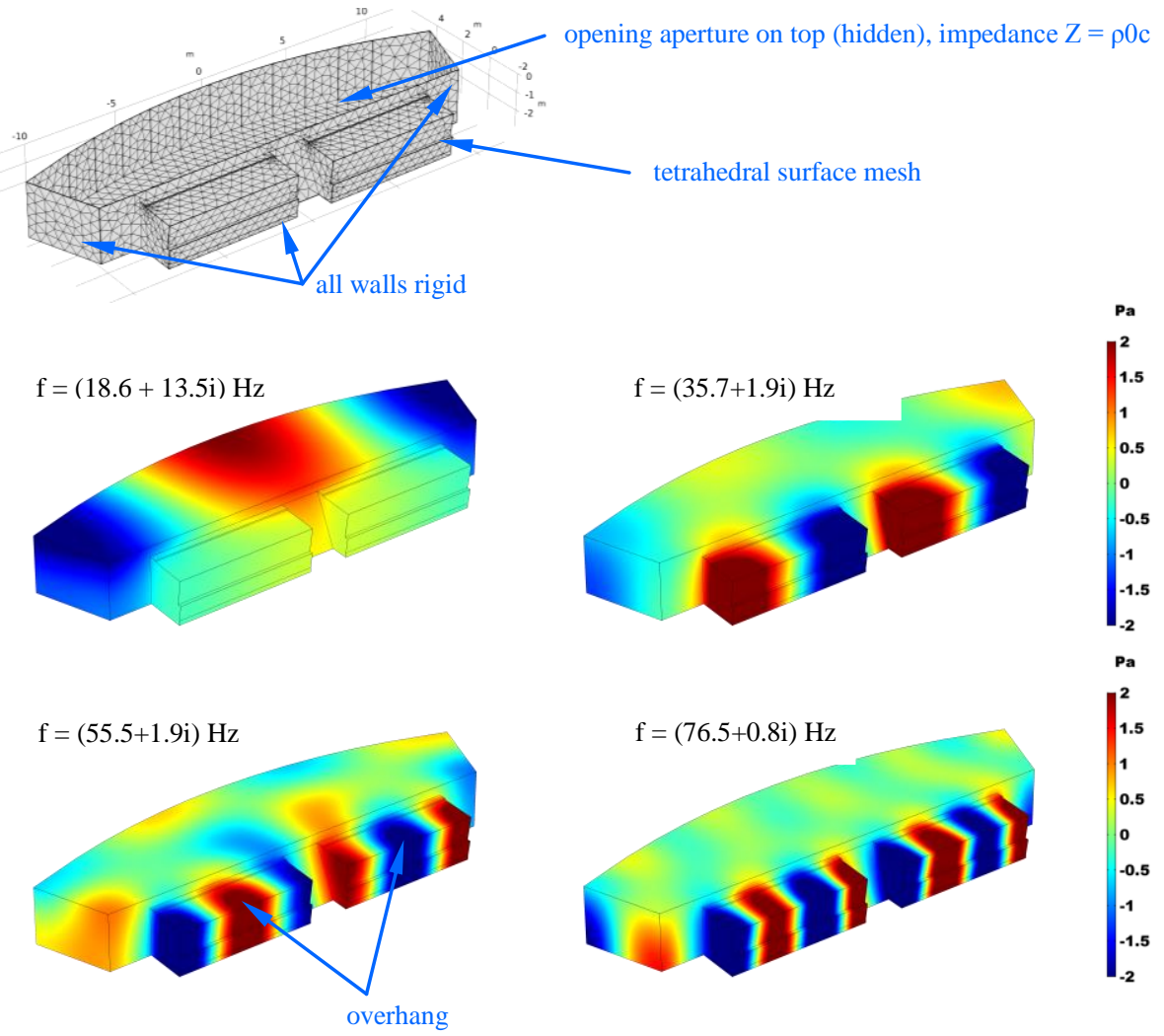

Fig. 3. Top: Meshed orchestra pit with meshing constraint $1_{\text {mesh }} \leq \lambda / 6$, opening aperture hidden (for clarity, only surface mesh shown here).

Below: Sound pressure in $\mathrm{Pa}$ of the orchestra pit for chosen eigenmodes \& natural frequencies. Please note the high sound pressures in the overhang area under the stage.

In the next step a simplified model of the auditorium together with the coupled orchestra pit is investigated by means of FEM in order to visualize the eigenmodes as shown in Fig. 4. The iron curtain is closed and the curtain itself and the ceiling are hidden in the plot for reasons of visibility. Here, the magnitude of the sound pressures is given at the surfaces at a natural frequency of $31.7 \mathrm{~Hz}$. Although the real part of sound pressure is shown in Fig.3 and the magnitude of sound pressure in Fig. 4 the similarity between the results is obvious 
(pl. compare Fig. 3 and Fig.4). Furthermore the relatively high sound pressures in the orchestra pit, in particular at the overhang, are clearly visible.

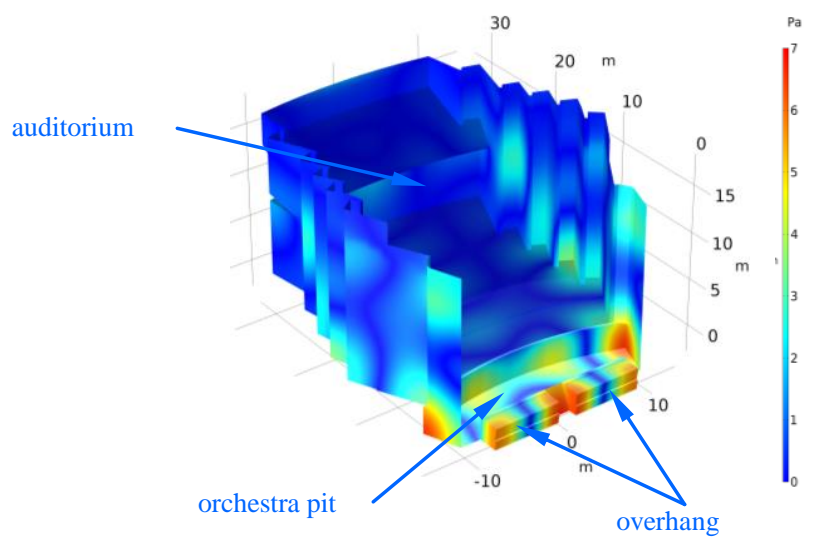

Fig. 4 Eigenmode for the idealized orchestra pit and auditorium (at natural frequency $f=31.7 \mathrm{~Hz}$ ). The ceiling and the iron curtain are hidden. Shown is the magnitude of the sound pressure in Pa.

\subsection{Calculations with Geometrical Acoustics}

In order to investigate the room acoustics for frequencies considerably higher than the Schroeder frequency, it is advantageous to use the methods of Geometrical Acoustics. For geometrical acoustics simulations a simplified model of the DOB has been set up. In order to match the reverberation time $\mathrm{T}_{30}$ in the CATT-Acoustic model (CATT-Acoustic v9.1c TUCT2 v2), first the given equivalent absorption area data in [2] was inserted. The derived absorption coefficients had then to be adjusted in order to obtain the measured $\mathrm{T}_{30}$ values as given in Fig. 2 with closed iron curtain. Auto edge scattering is enabled and a default surface diffuse reflection coefficient of 0.1 is applied to all surfaces except for the audience planes. For these planes separate coefficients have been used. Furthermore, the model can be used for estimating the influence of acoustic treatment and constructional changes in the orchestra pit on acoustics in the auditorium. A first study was carried out by modeling the influence of low frequency absorption in the orchestra pit. As a limiting case, all of the surfaces inside the pit were covered with low frequency absorbers in the model. In Fig. 4, the effect of low frequency absorption treatment on sound strength $G$ for the audience planes in the stalls and the galleries is displayed. The calculated reduction of sound strength $\mathrm{G}$ is of the order of $3 \mathrm{~dB}$. Inside the orchestra pit the reduction of the summarized sound strength $\mathrm{G}$ is of the order $5 \mathrm{~dB}$ as shown in Fig. 6 . Since sound strength $\mathrm{G}$ is related to the perceived loudness, this room acoustic parameter is relevant for evaluating the effect of acoustic treatment besides the sound pressure level, which is relevant for legal terms regarding health and safety. Since the Schroeder frequency of the decoupled orchestra pit is approximately around $100 \mathrm{~Hz}$, results of Geometrical Acoustic simulations should be critically evaluated here. For this case, FEM calculations shall be carried out later for comparing them with the results obtained by geometrical acoustics simulations. The results presented here have to be considered as a limiting case and a first test of the simulation model since the implementation of absorbers all around the orchestra pit is not a realistic measure. 

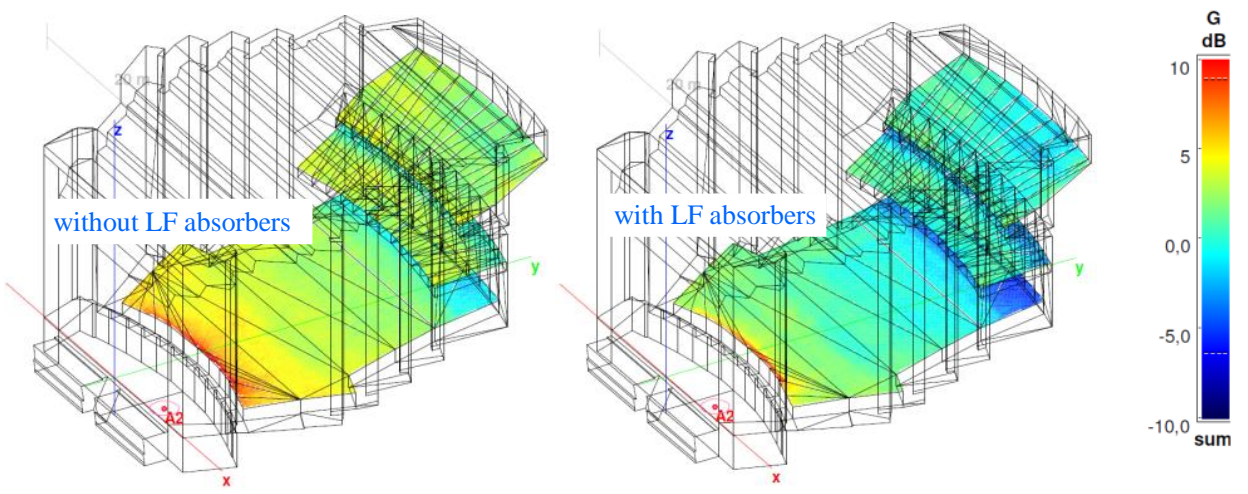

Fig. 5 Sound strength $G$ for a source A2 inside the orchestra pit for the current state (left) and with low frequency absorbers inside the orchestra pit (right). The calculated reduction of sound strength $\mathrm{G}$ is of the order of $3 \mathrm{~dB}$ (CATT-Acoustic, 962950 rays, $\mathrm{t}=1638 \mathrm{~ms}$ (left), $\mathrm{t}=1185 \mathrm{~ms}$ (right)).
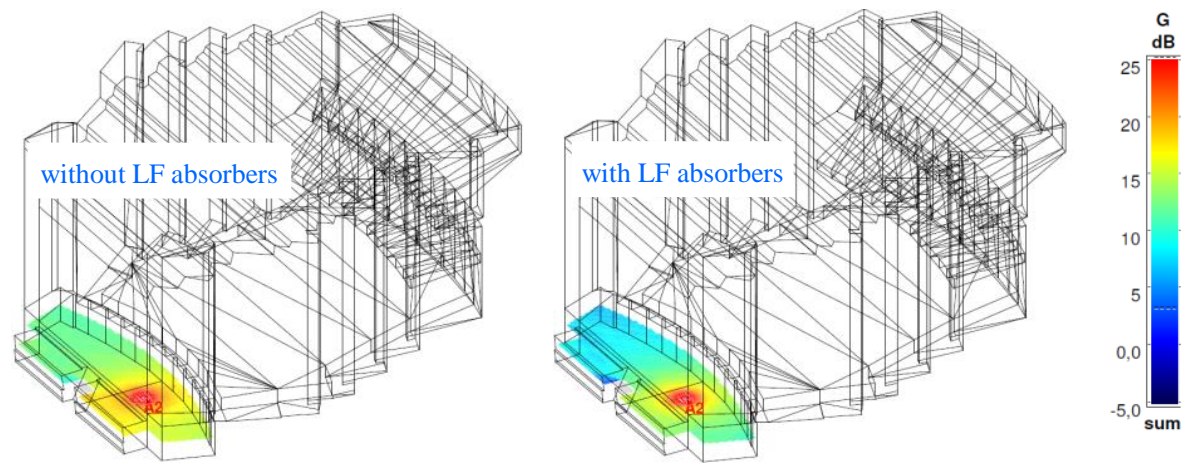

Fig. 6 Change in sound strength $G$ inside the orchestra pit for the current state on the left and with low frequency (LF) absorbers inside the orchestra pit. The reduction of sound strength inside the orchestra pit is in the order of $5 \mathrm{~dB}$ (Simulated with CATT-Acoustic v9.1c TUCT2 v2.962950 rays, $\mathrm{t}=1643 \mathrm{~ms}$ (left), $\mathrm{t}=1112 \mathrm{~ms}$ (right)).

\section{Summary and Conclusions}

The orchestra pits in opera houses pose a significant problem, since the sound pressure levels may exceed those thresholds that can be regarded as safe for the health of orchestra musicians. In order to provide the necessary data base for the evaluation of simulation results, room acoustic parameters such as reverberation time, clarity or strength have been determined for the Deutsche Oper Berlin. The characteristic values comply well with the established standards and are comparable to other opera houses. The reverberation time shows a strong dependency on both the frequency and on the experimental setup, e.g. with the iron curtain open or closed. Subsequently, two different methods of room acoustic simulations have been performed: The wave based Finite Element Method (FEM) and the geometrical acoustic method of Ray Tracing. First of all, FEM simulations have been performed for the discrete, i.e. decoupled orchestra pit. Several eigenmodes have been detected, exhibiting a strong dependency of sound pressure levels on both the frequency and the location, e.g. in the overhang of the orchestra pit. In addition, first results of the orchestra pit coupled to the auditorium are presented here. It is demonstrated, that the 
highest sound pressure levels for low frequencies can be found in the orchestra pit exclusively.

The application of Ray Tracing methods for middle and high frequencies requires significantly less computational power. Hence, it is possible to determine the room acoustic parameters, such a strength $G$, for the auditorium and the coupled orchestra pit. Now, additional measures to control the sound pressure levels can be investigated, such as the intensive use of absorbers in the orchestra pit. The so determined results can be thoroughly analysed and compared to the original set-up. In the future, it would be beneficial to compare simulation results of both the FEM and the Ray Tracing Method for the same frequencies, e.g. in the middle frequency range. At this stage, the performed measurements and simulation results will help for the investigation and evaluation of suitable measures in order to decrease sound pressure levels in the orchestra pit, support the mutual hearing of the orchestra musicians while maintaining the present acoustics in the auditorium.

The authors gratefully acknowledge the support by the project partners Deutsche Oper Berlin and wax $\mathrm{GmbH}$ as well as the research funding by Institut Für Angewandte Forschung (IFAF) Berlin.

\section{References}

1. M. Barron, Auditorium Acoustics and Architectural Design, $2^{\text {nd }}$ ed. (Spon Press, London and New York, 2010)

2. L. Cremer, J. Nutsch, H. J. Zemke, "Die akustischen Maßnahmen beim Wiederaufbau der Deutschen Oper Berlin", Acustica, 12, 428 - 433 (1962)

3. L. Cremer, H. A. Müller, Die wissenschaftlichen Grundlagen der Raumakustik - Band 1, $2^{\text {nd }}$ ed., (Hirzel Verlag, Stuttgart, 1978)

4. A. Schlesinger, M. Ochmann, J. M. Kimmich, S. Frank, "Controlling multiple acoustic objectives during the implementation of the European Directive 2003/10/EC at an opera house", Proc. of Euronoise `18, 2115 - 2122 (2018)

5. Acoustics - Measurement of room acoustic parameters - Part 1: Performance spaces, ISO 3382-1:2009 (2009)

6. H. Kuttruff, Room Acoustics, $5^{\text {th }}$ ed. (Spon Press, London and New York, 2009)

7. A. C. Gade, "Acoustics in Halls for Speech and Music", in Handbook of Acoustics, T. D. Rossing, Ed. (Springer, New York, 2007)

8. A. C. Gade, "Acoustics for symphony orchestras; status after three decades of experimental research", Proc. Of the ISRA `10 (2010)

9. J. J. Dammerud, "Stage Acoustics for Orchestras in Concert Halls", PhD thesis, University of Bath (2009)

10. M. Vercammen, M. Lautenbach, "Stage and pit acoustics in opera houses", Proc. of the ISRA `16 (2016)

11. R. Tomiku, S. Sakamoto, N. Okamoto, Y. Yasuda, Y. Horinouchi, K. Hoshi, "Room Acoustics Simulation", in Comp. Sim. in Arch. \& Env. Ac. (Springer, Tokyo, 2014)

12. Y.H. Wijnant, E.R. Kuipers, A. de Boer, "Development \& applic. of a new method for in-situ measurement of sound absorption", Proc. ISRA 10109 - 122 (2010)

13. M. Aretz, "Combined Wave and Ray Based Room Acoustic Simulations of Small Rooms", (Logos Verlag, Berlin, 2013) 